\title{
(1) \\ O Legado Didático da Convergência de Interface Tangível com Realidade Aumentada no Magistério de Cartografia Tridimensional
}

\author{
Laura Quevedo Jurgina $^{1}$, Domarys Corrêa ${ }^{1}$, Gabriella Selbach ${ }^{1}$, \\ Felipe de Souza Marques ${ }^{1}$, Leomar Soares da Rosa Junior ${ }^{1}$ \\ ${ }^{1}$ Centro de Desenvolvimento Tecnológico (CDTec) \\ Universidade Federal de Pelotas (UFPel) - Pelotas, RS - Brasil
}

\begin{abstract}
This work presents an evaluation of an educational tool that uses Augmented Reality and Tangible Interface to allow undergraduate students to efficiently learn topography and 3D cartography. The work was carried out by PET-Computação targeting students from Computer Science and Hydraulic Engineering courses at the Universidade Federal de Pelotas. The results show the tool feasibility, which is still barely explored in Brazilian universities. Furthermore, the study notes that the use of Augmented Reality and Tangible Interface is well accepted by the academic community and can serve as an aid technology to traditional teaching-learning methods.
\end{abstract}

Resumo. Este trabalho apresenta a avaliação de uma ferramenta educacional que utiliza Realidade Aumentada e Interface Tangível para auxiliar os alunos, de curso superior, na compreensão mais eficiente dos tópicos das disciplinas que abordam topografia e cartografia tridimensional. $O$ trabalho foi realizado como uma ação do Grupo PET-Computação, tendo como público alvo alunos dos cursos de Computação e de Engenharia Hídrica da Universidade Federal de Pelotas. Os resultados alcançados demonstram a viabilidade de aplicação da ferramenta, a qual ainda é pouco explorada nas universidades brasileiras. Ainda, o estudo constata que a utilização de Realidade Aumentada e Interface Tangível é bem aceita pela comunidade acadêmica, podendo servir como tecnologia de auxílio aos tradicionais métodos de ensino-aprendizagem.

\section{Introdução}

No ensino, a utilização de recursos computacionais precisa estar comprometida com métodos que possibilitem a concepção sobre o conhecimento. As ferramentas devem despertar a reflexão sobre o seu auto aprendizado, incentivando, desta maneira, a recepção solícita ao tema (LOPES; SILVA; ALMEIDA, 2012).

Atualmente, existem diversas ferramentas computacionais aplicadas no Ensino Básico e no Ensino Superior. As aplicações podem ser encontradas facilmente na Web. Para o aprendizado de matemática no Ensino Médio, dentre vários softwares disponíveis, existe o emprego de MatLab perante - por exemplo - visualização de gráficos de funções (VIEIRA; MORAIS, 2013). No Ensino Superior, ferramentas de auxílio a aprendizagem, tais como o SwicthCraft (CALLEGARO, 2010) e o Karma (KLOCK; RIBAS; REIS, 2010), são utilizadas para exercitar os conteúdos vistos por alunos de disciplinas de Técnicas e de Circuitos Digitais em cursos de Engenharia e de Computação. Outra 
modalidade que também vem crescendo é a adoção do uso de dispositivos móveis por alunos do Ensino Médio, onde existem aplicativos para acesso de questões preparatórias para vestibulares (EIT, 2019) (PRATES, 2019). Da mesma forma, no Ensino Superior, aplicativos para smartphones vêm sendo adotados em disciplinas que exploram o uso de tecnologias em suas grades curriculares. O Circuit Scramble (SUBORBITAL, 2019) e o Pythagorea (HORIS, 2019) são exemplos de ferramentas utilizadas para este fim.

Recursos computacionais como Realidade Aumentada (RA) e Interfaces Tangíveis (TUI) têm sido empregadas em diversas ferramentas eletrônicas e áreas do conhecimento. Isso se deve ao fato destes recursos permitirem que o usuário interaja de maneira imersiva no problema a ser estudado ou resolvido (DE LIMA, 2017) (DOS REIS; GONÇALVES, 2016).

A RA configura-se como uma modalidade de interface computacional avançada que busca alcançar a interação humano computador de uma forma mais natural e que mistura, em tempo real, objetos virtuais gerados por computador com elementos do ambiente físico. Os objetos virtuais são visualizados por meio da utilização de dispositivos tecnológicos de saída de dados, tais como óculos especiais (head mounted displays), smartphones e projetores, produzindo um ambiente único com impressão de continuidade ao usuário (COLPANI; HOMEM, 2016).

Segundo DE LIMA (2017), temos um ambiente de realidade aumentada quando filmamos um local, em tempo real, e inserimos objetos virtuais, onde as cenas formadas dão a impressão de que os objetos virtuais pertencem ao mundo real. Um exemplo icônico de RA é o famoso aplicativo Pokémon GO, onde o usuário, utilizando um dispositivo móvel, encontra personagens da série no ambiente real (PADRÃO, 2016), como ilustrado na Figura 1.

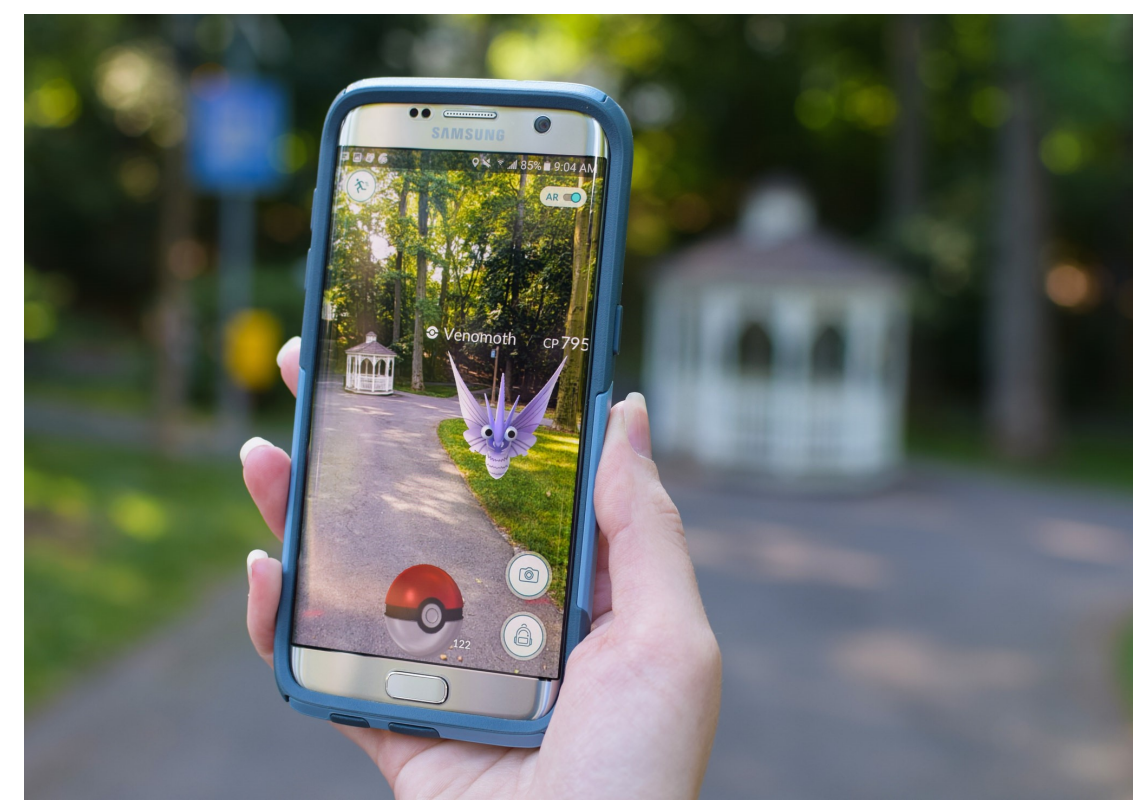

Figura 1. Realidade aumentada em aplicativo móvel (NIANTIC, 2019).

Com o avanço das tecnologias computacionais, especialmente àquelas relacionadas com a interação do usuário e o sistema digital, um novo campo de estudos se desen- 
volveu na computação, dando início às Interfaces Tangíveis. As TUI podem ser definidas como interfaces que compreendem interações realizadas em artefatos físicos, como estímulos para interferir no contexto e representações de informação digital. Simuladores veiculares são um exemplo de aplicação de Interfaces Tangíveis, uma vez que envolvem o uso de objetos físicos para manipulação de cenários virtuais (DOS REIS; GONÇALVES, 2016). A Figura 2 ilustra este conceito.

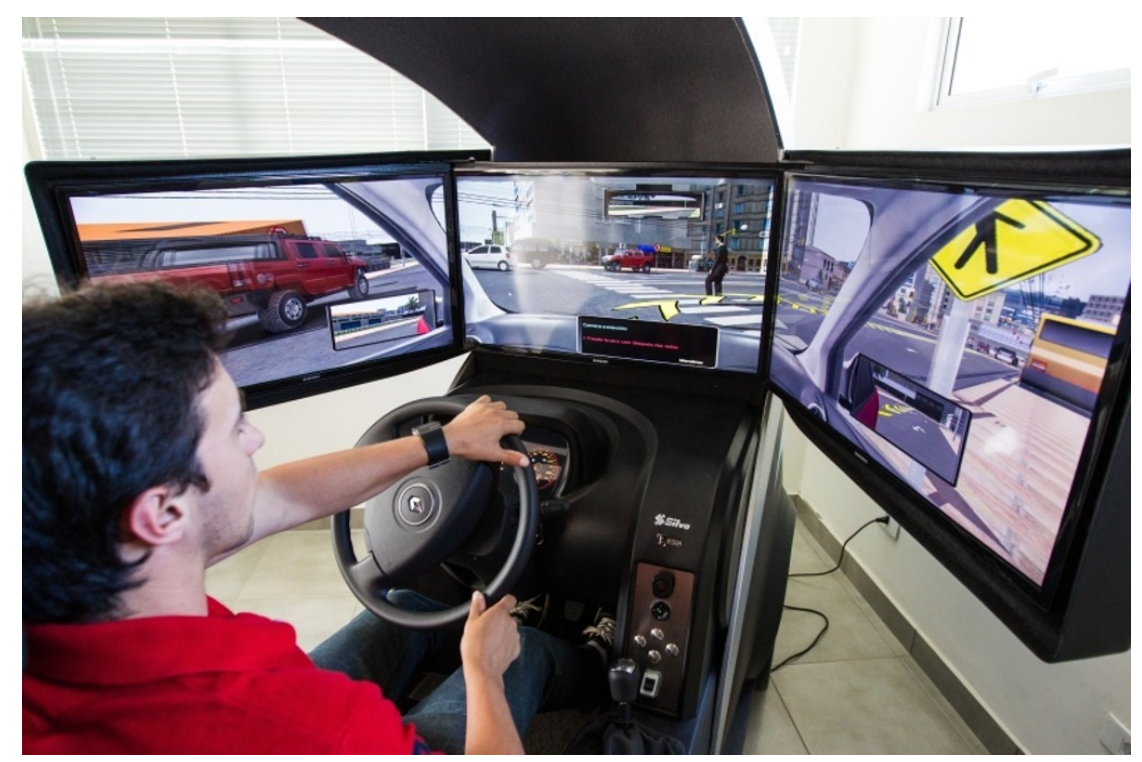

Figura 2. Simulador veicular utilizando Interface Tangível (GAZETA, 2016).

Existem trabalhos que debatem sobre a inserção Realidade Aumentada no contexto de ensino para auxílio do aprendizado. WU (2013) e EL SAYED (2011) discutem sobre métodos para o sucesso da tecnologia como instrumento pedagógico. DOS REIS (2016) discute, a partir de revisões literárias, sobre o conceito e a usabilidade de interfaces tangíveis.

Neste contexto, este artigo tem o intuito de avaliar uma ferramenta educacional desenvolvida para estudos topográficos, investigando, assim, a eficácia do seu emprego no aprendizado. Esta ferramenta faz uso de Realidade Aumentada e de Interface Tangível para permitir que os educandos consigam compreender de forma mais eficiente os conteúdos ensinados nas disciplinas que abordam topografia e cartografia tridimensional. A seção 2 deste trabalho apresenta a metodologia adotada. Na seção 3 os resultados obtidos são apresentados. Por fim, a seção 4 apresenta a discussão final e as conclusões.

\section{Metodologia}

A metodologia de pesquisa utilizada neste trabalho é conhecida e classificada como "pesquisa com survey". Segundo GERHARDT e SILVEIRA (2009) "pesquisa com survey" é o estudo que busca informação diretamente com uma comunidade de interesse a respeito dos dados que se deseja obter. Dessa forma, utilizamos esse instrumento para quantificar as opiniões de um grupo de pessoas representantes do nosso público-alvo, estudantes universitários de Computação e de Engenharia Hídrica, sobre o objeto da pesquisa: a ARSandBox. Por fim, um questionário eletrônico foi utilizado para coletar as respostas. 
A ARSandBox consiste em uma aplicação que integra Realidade Aumentada e Interface Tangível para visualização 3D de ambiente geográfico, através de mapa topográfico (DARLEY, 2017). A disposição da ARSandBox inclui uma caixa de areia com área de 1,5 m2; sensor Kinect 3D e projetor, localizados a $1 \mathrm{~m}$ de altura; um computador equipado com qualquer sistema operacional Linux, $2 \mathrm{~Gb}$ de memória RAM e placa gráfica Nvidia para executar o software que realiza a decodificação da área de interação (UCDAVIS, 2019). A Figura 3 apresenta o esquemático do sistema da ARSandBox. Seu funcionamento acontece por meio da manipulação da areia (TUI), recebendo a modificação estrutural dos usuários para formar relevos. O sistema capta a modificação física realizada e interpreta as novas curvas de níveis formadas, projetando as cores adequadas na areia. A Figura 4 ilustra a utilização da ARSandBox.

Este projeto foi implantado na UFPel através de uma iniciativa conjunta dos cursos de Ciência da Computação e de Engenharia Hídrica. Segundo DARLEY (2017), a grande dificuldade em ensinar topografia para os alunos é a limitação que a superfície plana tem de representar os relevos de um terreno. Em uma folha de papel ou em um quadro só é possível desenhar em duas dimensões. A aplicação da ARSandBox contorna esta limitação, oferecendo a noção de um modelo tridimensional que aprimora o entendimento do aluno. O mais interessante é que ela consiste em uma TUI, onde o aluno pode moldar a superfície, permitindo novas descobertas (DARLEY, 2017). A ARSandBox foi disponibilizada aos alunos da UFPel. Estes, então, foram convidados a experimentar seu funcionamento.

A interação do público com a caixa fora inicialmente livre, com movimentos e contatos espontâneos para que os alunos conhecessem o ambiente. Após o período de reconhecimento algumas ações foram estimuladas, tais como simular chuva e construir relevos de tamanhos diversos com o objetivo de demonstrar as funcionalidades da aplicação. Após a experiência, um questionário eletrônico de avaliação foi fornecido aos usuários para coletar suas percepções relativas ao uso da ferramenta.

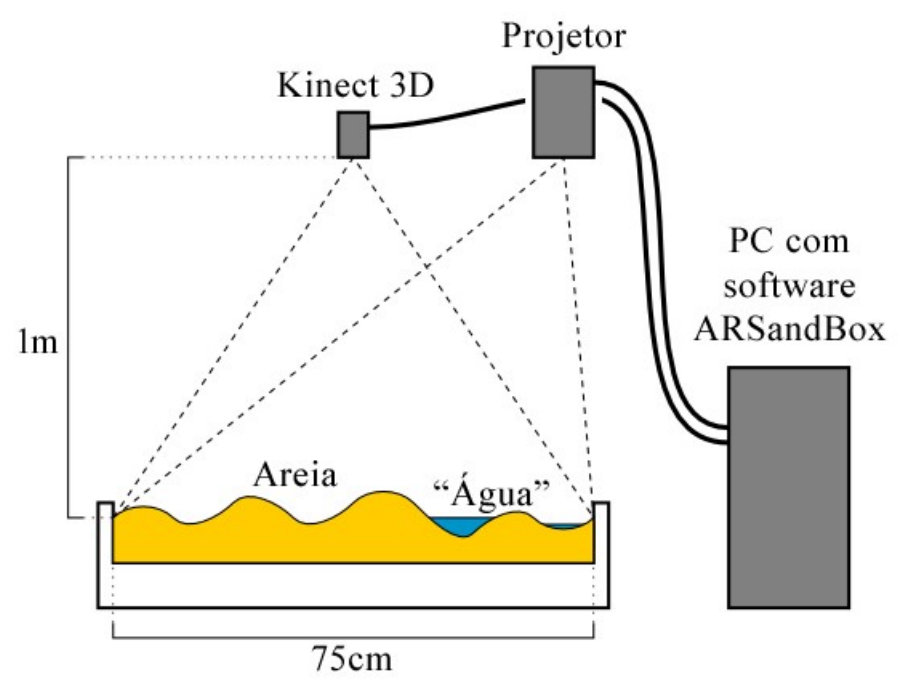

Figura 3. Arranjo típico do sistema da ARSandBox (UCDAVIS, 2019). 


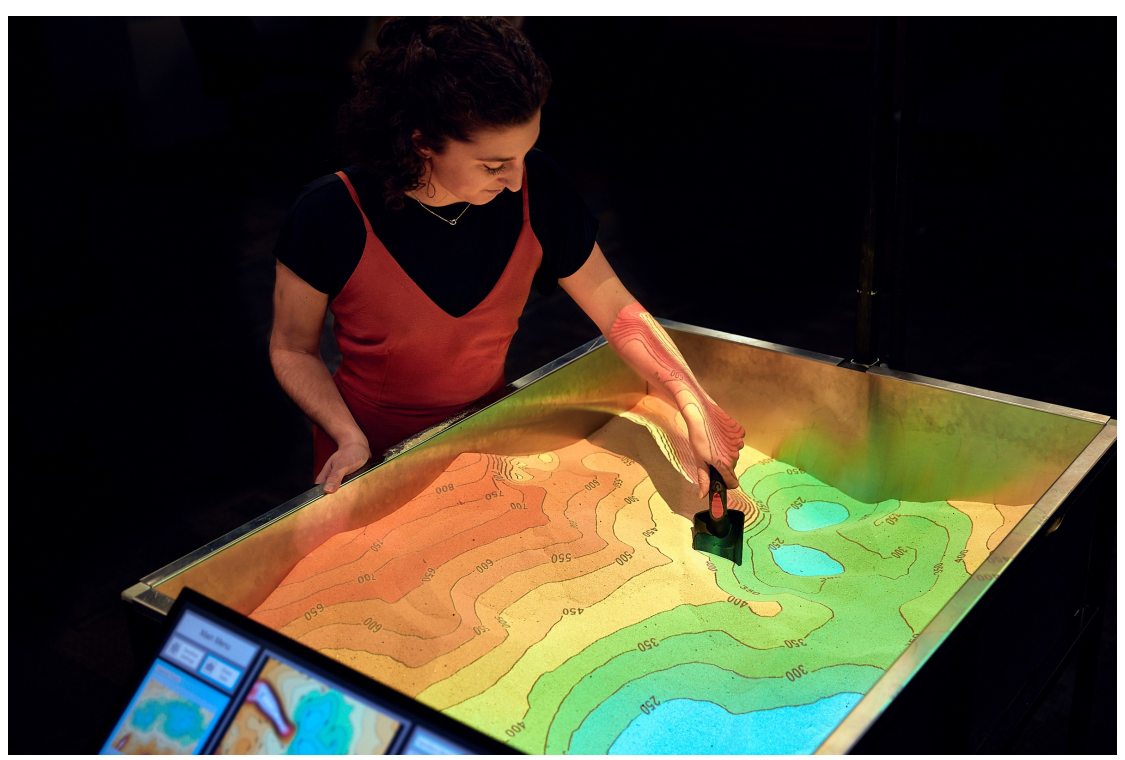

Figura 4. ARSandBox sendo utilizada para formar relevos.

\section{Resultados e Discussão}

Considerando a interatividade do público com a ARSandBox, em pesquisa realizada durante o segundo semestre de 2018 com alunos dos cursos de Computação e de Engenharia Hídrica da Universidade Federal de Pelotas, as seguintes perguntas foram realizadas:

1) "Se você utilizou a ARSandBox, que nota daria para sua experiência?";

2) "Você conseguiu utilizar a ferramenta com facilidade?";

3) "Você compreendeu facilmente o conteúdo com a ferramenta?";

4) "Você considera que o uso de RA e TUI para o ensino de outros conteúdos seria útil em sua formação acadêmica?";

5) "Você já havia utilizado alguma ferramenta de ensino com essas tecnologias?".

As avaliações dos usuários consideraram notas entre 0 e 10, sendo 0 para uma experiência ruim e 10 para uma experiência excelente. Ao todo, 150 alunos testaram a ARSandBox e responderam o questionário. O resultado da pesquisa é apresentado nas figuras abaixo.

De modo geral, os alunos relataram uma excelente experiência de uso. Dos 150 usuários da aplicação, 145 deram nota máxima para a experiência com a ARSandBox. Outros 5 alunos avaliaram a experiência com notas 8 e 9, o que ainda assim demonstra que a experiência com a Realidade Aumentada e a Interface Tangível é avaliada de maneira bastante positiva pelos usuários.

Em relação a facilidade de uso da ferramenta, podemos constatar que a maioria dos usuários, 145 alunos, avaliaram com nota máxima o uso da aplicação. Neste quesito, novamente, 5 alunos encontraram alguma dificuldade na utilização da ARSandBox. Embora ela seja considerada de fácil manuseio pela grande maioria dos usuários, é possível perceber que existe necessidade de ajustes e adaptações de modo a permitir que todos os usuários consigam utilizar a aplicação sem complicações. 


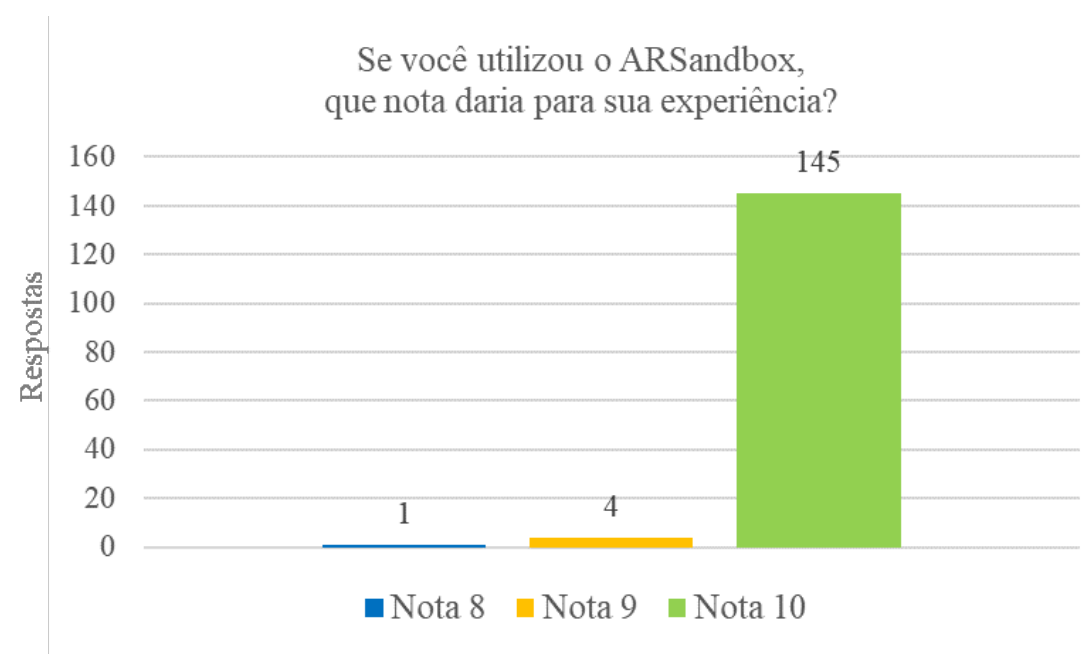

Figura 5. Avaliação da pergunta 1.

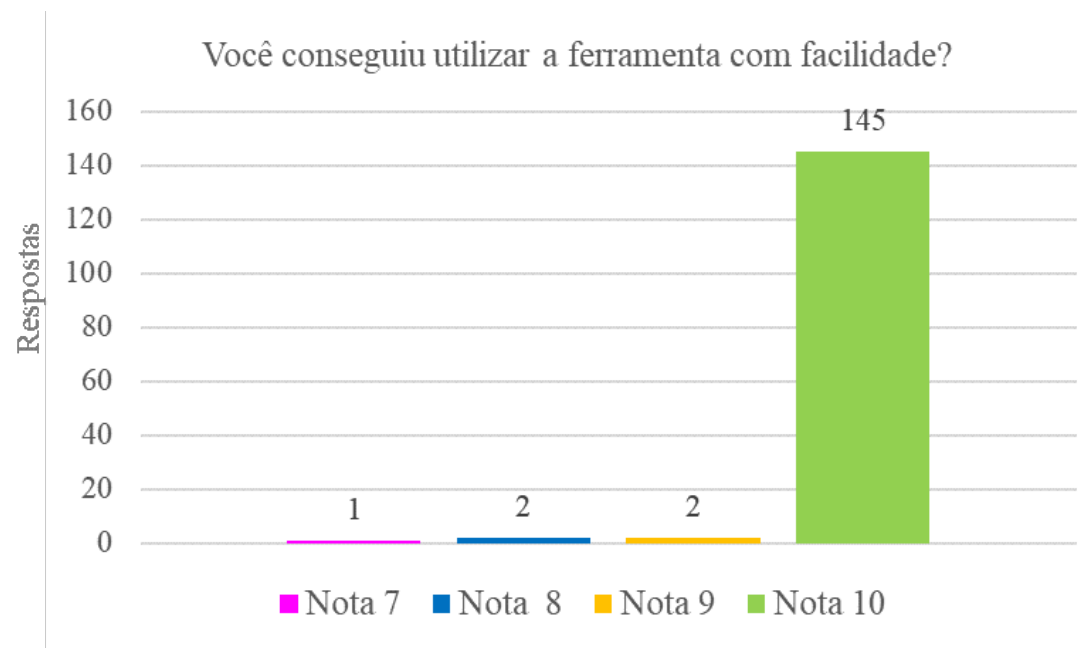

Figura 6. Avaliação da pergunta 2.

O conteúdo a ser ensinado com a utilização da ARSandBox foi compreendido por todos os usuários. Isto demonstra que a ferramenta cumpre o seu papel no ensino do conteúdo para o qual ela foi proposta. Esta avaliação corrobora com outros estudos disponíveis na literatura que afirmam que o uso de novas tecnologias pode trazer ganhos ao processo de ensino e aprendizagem, uma vez que a utilização de novas técnicas e ferramentas faz com que os alunos demonstrem um maior interesse pelos conteúdos apresentados.

Dos 150 alunos que utilizaram a ARSandBox e experimentaram o uso da Realidade Aumentada e da Interface Tangível, 141 responderam que estas tecnologias seriam úteis para a aprendizagem de outros conteúdos em suas formações acadêmicas. Apenas 9 usuários avaliaram com nota inferior. Embora não questionados nesta pesquisa sobre o motivo de terem avaliado com nota inferior, acredita-se que o motivo para tal se deve a dificuldade de alguns usuários em perceber a aplicação da RA e da TUI para o ensino de outros objetos de conhecimento. 


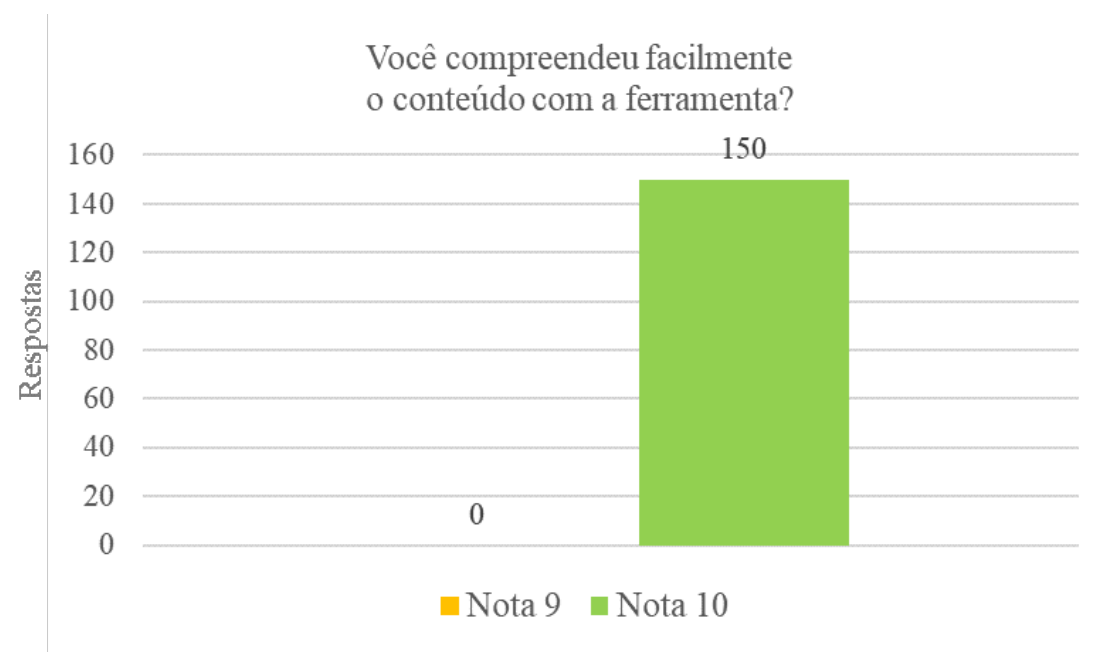

Figura 7. Avaliação da pergunta 3.

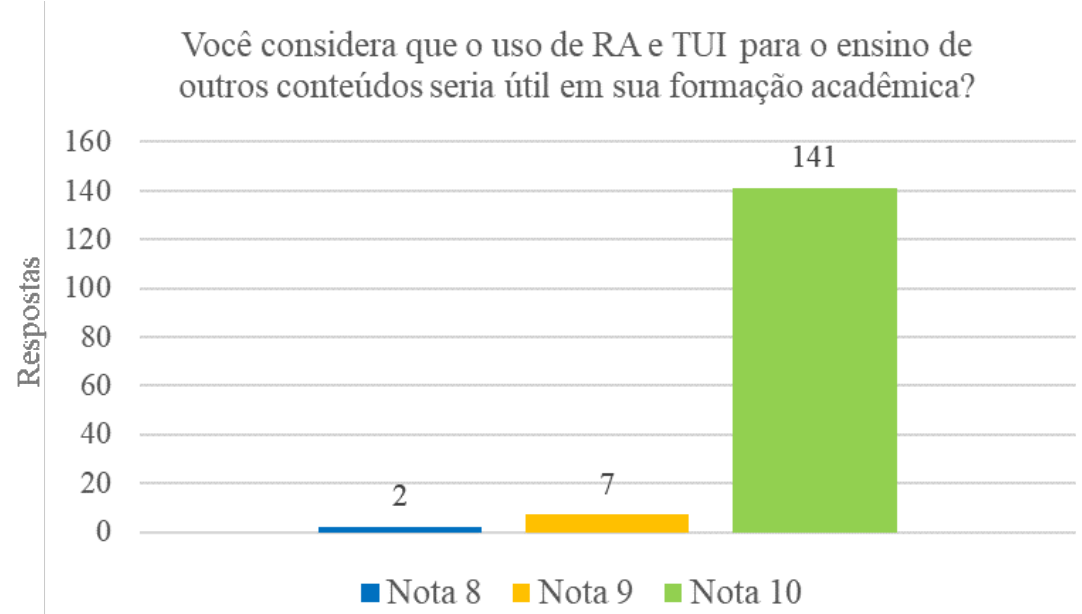

Figura 8. Avaliação da pergunta 4.

Por fim, com a última pergunta da avaliação, constata-se que a grande maioria dos usuários, 148 alunos, jamais haviam utilizado uma ferramenta que fizesse uso de Realidade Aumentada e de Interface Tangível em suas experiências acadêmicas. Este dado é interessante quando analisado junto às demais questões avaliadas por este trabalho. É possível perceber uma alta aceitação, interesse e facilidade dos alunos no uso de ferramentas com estas tecnologias. Porém, elas ainda são pouco exploradas no meio acadêmico. Mesmo em cursos de tecnologia, como Ciência da Computação, Engenharia de Computação, e Engenharia Hídrica, tais ferramentas que utilizam recursos imersivos de RA e TUI ainda são raramente empregadas no ensino. Isto se estende para outros cursos, tais como Engenharia Civil e Arquitetura e Urbanismo. Estes dois últimos, por exemplo, poderiam fazer uso da ARSandBox para cobrir conteúdos curriculares de uma maneira mais atrativa, auxiliando as técnicas atuais pelas quais o ensino é transmitido aos alunos. 


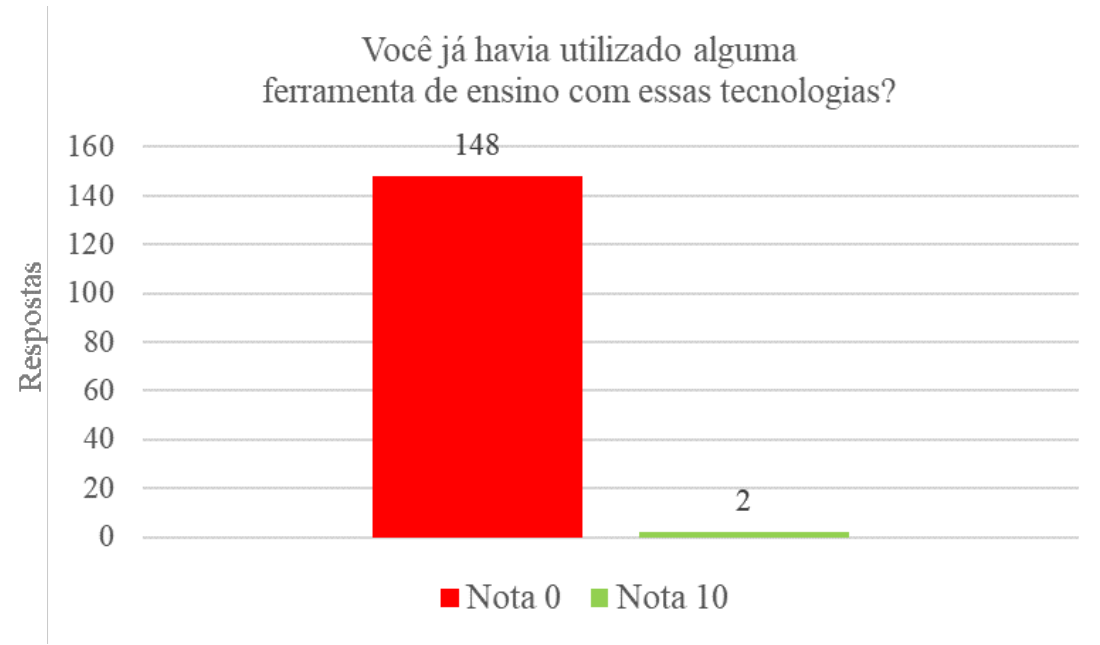

Figura 9. Avaliação da pergunta 5.

\section{Conclusões}

A partir das respostas positivas dos usuários à experiência com a ARSandBox, pode-se considerar a ferramenta um método diferencial no ensino de topografia. O uso combinado desta prática com o material 2D - já utilizado na teoria - faz com que ambas se complementem, atingindo todo o potencial de aprendizado.

Com o avanço da tecnologia, o emprego da mesma na simplificação de atividades torna-se inevitável. A RA não pode ser visualizada apenas como avanço utilizado em um mundo gamer, porém sim como ferramenta empregável diariamente. No meio educacional as vantagens oferecidas pela mescla de realidades integradas a uma interface tangível trazem uma colaboração visual e sensível na compreensão de projetos em 3D que são abstratos quando no plano.

\section{Agradecimentos}

Os autores agradecem o Programa de Educação Tutorial do Ministério da Educação e as agências de fomento CNPq e FAPERGS pelo apoio no desenvolvimento deste trabalho.

\section{Referências}

LOPES, M. P.; SILVA, R. B.; ALMEIDA, A. O. A importância do uso das ferramentas computacionais no ensino da disciplina fenômenos de transportes nos cursos de engenharia. In: ANAIS DO CONGRESSO BRASILEIRO DE EDUCAÇÃO EM ENGENHARIA, 2012. v.XL. p.103.

VIEIRA, C.; MORAIS V. MATLAB, Curso Completo. FCA. $1^{\text {a }}$ edição. 2013.

CALLEGARO, V.; MARQUES, F. S.; KLOCK, C. E.; DA ROSA JR, L. S.; RIBAS, R. P.; REIS, A. I. SwitchCraft. In: 23rd Symposium on Integrated Circuits and System Design, 2010.

KLOCK, C. E.; RIBAS, R. P.; REIS, A. I. Karma: um ambiente para o aprendizado de síntese de funções Booleanas. Revista Brasileira de Informática na Educação, v.18, p.3342, 2010. 
EIT. AppProva ENEM 2018 - Simulados e Questões Inéditas. Apple Store, 30 mar. 2019. Acessado em 30 mar. 2019. Online. Disponível em: https://itunes.apple.com/br/app/appprova-enem-2018/id639910973

PRATES, L. Studos - Simulados ENEM e Vestibulares. Apple Store, 30 mar. 2019. Acessado em 30 mar. 2019. Online. Disponível em: https://itunes.apple.com/br/app/studos/id819499553

SUBORBITAL, G. Circuit Scramble. Google Play, 30 mar. 2019. Acessado em 30 mar. 2019. Online. Disponível em: https://play.google.com/store/apps/developer?id=Suborbital+Games

HORIS I. L. Pythagorea. Google Play, 30 mar. 2019. Acessado em 30 mar. 2019. Online. Disponível em: https://play.google.com/store/apps/details?id=com.hil ${ }_{h} k$.pythagorea

DE LIMA, A. L. F. et al. A importância da realidade aumentada aplicada à educação. In: IV CONGRESSO NACIONAL DE EDUCAÇÃO, 2017.

DOS REIS, A. V.; GONÇALVES, B. S. Interfaces Tangíveis: Conceituação e Avaliação. Estudos em Design, Rio de Janeiro, v.24, n.2, p.92-111, 2016.

COLPANI, R.; HOMEM, M. R. P. Realidade Aumentada e Gamificação na Educação: uma aplicação para auxiliar no processo de aprendizagem de alunos com deficiência intelectual. São Carlos: UFSCar, Revista Brasileira de Informática na Educação, 2016.

PADRÃO, M. Entenda a realidade aumentada, recurso por trás do sucesso de Pokémon Go. UOL, São Paulo, 05 ago. 2016. Acessado em 29 mar. 2019. Online. Disponível em: https://tecnologia.uol.com.br/noticias/redacao/2016/08/05/entendaarealidade-aumentada-recurso-por-tras-do-sucesso-de-pokemon-go.htm

NIANTIC. Pokémon GO. The Pokémon Company International, Inc. Acessado em 29 mar. 2019. Online. Disponível em: https://www.pokemon.com/us/pokemon-videogames/pokemon-go/

DOS REIS, A. V. Interfaces Tangíveis em Simuladores Veiculares: Componentes para um Protocolo de Avaliação de Usabilidade. 2016. 129f. Dissertação (mestrado) - Programa de Pós-Graduação em Design e Expressão Gráfica, Universidade Federal de Santa Catarina.

GAZETA. Autoescolas de Araguari incluem simuladores de direção veicular em suas aulas. Gazeta do Triângulo, Araguari, Minas Gerais, 11 nov. 2016. Acessado em 29 mar. 2019. Online. Disponível em: http://gazetadotriangulo.com.br/tmp/noticias/autoescolasde-araguari-incluem-simuladores-de-direcao-veicular-em-suas-aulas/

GERHARDT, T. E.; SILVEIRA, D. T. Métodos de pesquisa. Editora da UFRGS. $1^{\mathrm{a}}$ edição. 2009.

DARLEY, N. T.; et al. Tangible Interfaces: An Analysis of User Experience Using the ARSandbox Project. In: SIMPÓSIO BRASILEIRO SOBRE FATORES HUMANOS EM SISTEMAS COMPUTACIONAIS, 2017.

UCDAVIS. Augmented Reality Sandbox. UC Davis' W.M. Keck Center for Active Visualization in the Earth Sciences. Acessado em 14 apr. 2019. Online. Disponível em: https://arsandbox.ucdavis.edu/ 
WU, H.-K. et al. Current status, opportunities and challenges of augmented reality in education. Computers Education, Elsevier Ltd, mar. 2013.

EL SAYED, N. A. M. E.; ZAYED, H. H.; SHARAWY, M. I. Augmented reality student card. Computers Education, maio 2011. 